Proceedings of the XXIII Conference on Applied Crystallography, Krynica Zdrój, Poland, September 20-24, 2015

\title{
Mould Walls Inclination and Dendritic Morphology of CMSX-4 Blades Airfoils
}

\begin{abstract}
J. KRAWCZYK ${ }^{a, *}$, W. Bogdanowicz ${ }^{a}$, J. SieniaWski ${ }^{b}$ and K. KubiaK ${ }^{b}$
${ }^{a}$ Institute of Materials Science, University of Silesia, 75 Pułku Piechoty 1a, 41-500 Chorzów, Poland ${ }^{b}$ Department of Materials Science, Rzeszów University of Technology, W. Pola 2, 35-959 Rzeszów, Poland

The airfoils of single-crystalline turbine blades were studied. The blades made of industrial CMSX-4 superalloy were obtained by the Bridgman technique. Five different withdrawal rates $(1-5 \mathrm{~mm} / \mathrm{min})$ were used. Series of as-cast samples were prepared by cutting the airfoils at different distances from the blade root. The metallographic sections, prepared for each cutting planes, were subjected to scanning electron microscopy observations and Laue diffraction studies. Macrostructure images of whole area of airfoil cross-sections were obtained by stitching several obtained SEM images. Morphology of dendrites in different area of cross-sections were analysed. The crystal orientation of each sample were determined by analysis of Laue pattern. It was found that the morphology of dendrites changes in the area, where the dendrite growth is limited by inclined mould walls.
\end{abstract}

DOI: 10.12693/APhysPolA.130.1100

PACS/topics: 68.70.+w, 61.66.Dk, 61.72.Bb

\section{Introduction}

The single-crystalline superalloys are useful material in many industry sectors, inter alia, for the production of blades in gas turbines or aircraft engines. These hightemperature alloys have increased strength properties, especially heat and creep resistance at high operating temperatures. The conditions, in which the turbine blades operate, require specific strength properties, that possess CMSX-4 superalloy [1, 2]. Crystallization process of single-crystalline blades is difficult due to their complexity shape, and for this reason, microstructure disorders can be formed during the production. The blades geometry can affect the dendritic structure and crystal orientation, which is an important factor influencing the strength of the blades $[3,4]$. One of the important parameters in the crystallization of turbine blades is withdrawal rate [5]. The aim of these studies is to analyse the influence of the geometrical relationships between the dendrite growth direction and mould walls inclination on dendritic morphology in blades airfoil, leading to the improvement of blades durability.

\section{Material and investigations}

The turbine blades were produced by the Bridgman technique [6] with five different withdrawal rates of 1,2 , 3,4 and $5 \mathrm{~mm} / \mathrm{min}$. The blades were examined in as cast state. The specimens for investigations were cut parallel to the base plane (BP), which was perpendicular to the withdrawal axis $Z$ (Fig. 1). The surfaces of specimens were perpendicular to the axis $Z$. Three specimens from each blades airfoil were prepared. The specific angles $\delta^{L}$, $\delta^{T}$ (Fig. 1) of blades geometry, related to the leading edge (LE) and trailing edge (TE) for all specimens of all sections were determined. The $\delta^{L}, \delta^{T}$ angles are measured

\footnotetext{
*corresponding author; e-mail: jacek.krawczyk@us.edu.pl
}

between $Z$ and LE or TE in all sections. Measured angles value are: $\delta_{1}^{L}=8^{\circ}, \delta_{2}^{L}=12^{\circ}, \delta_{3}^{L}=12^{\circ}, \delta_{1}^{T}=5.5^{\circ}$, $\delta_{2}^{T}=4^{\circ}, \delta_{3}^{T}=1.5^{\circ}$.

The crystal orientation of specimens was analysed by back-reflection Laue method with the use of Co X-ray radiation. The microstructure of blades airfoil was analysed by scanning electron microscopy (backscattering electron) SEM (BSE) technique. The SEM macro-images were created by the merging of micrographs, made over the entire microsection surface with magnification of $25 \times$.
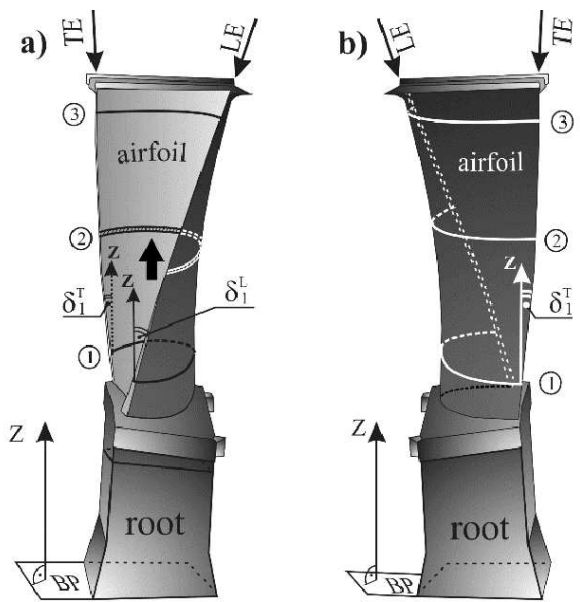

Fig. 1. Shape of the blade with marked section planes (1-3) and examples of $\delta_{1}^{L}, \delta_{1}^{T}$ angles of the (a) LE and (b) TE of section $1, \mathrm{BP}$ - base plane, $Z-$ withdrawal axis.

\section{Results and discussion}

Figure 2 shows example of SEM macro-image, obtained from whole sample by merging many separate SEM images. Typical dendritic structure is visible with dendrite arms arranged in lines. The dendrite arms are parallel to $\mathrm{a}$ and b lines (Fig. 2) and corresponds to the Laue 
spots arrangement in the Laue patterns (Fig. 3). The morphology of dendrites near suction and pressure surfaces is different (Fig. 2d, area F1,F2). In some areas of the sections, near the mould walls, the morphology of dendrites resembles a conifer tree, while in other areas is a typical morphology of the four-petals flower (Fig. 2d). The "tree-like" morphology is created by growth of tertiary dendrite arms out from single secondary dendrite arm (Fig. 2d, area F1) or by growth and one of the two multiplication systems of secondary dendrites arms.

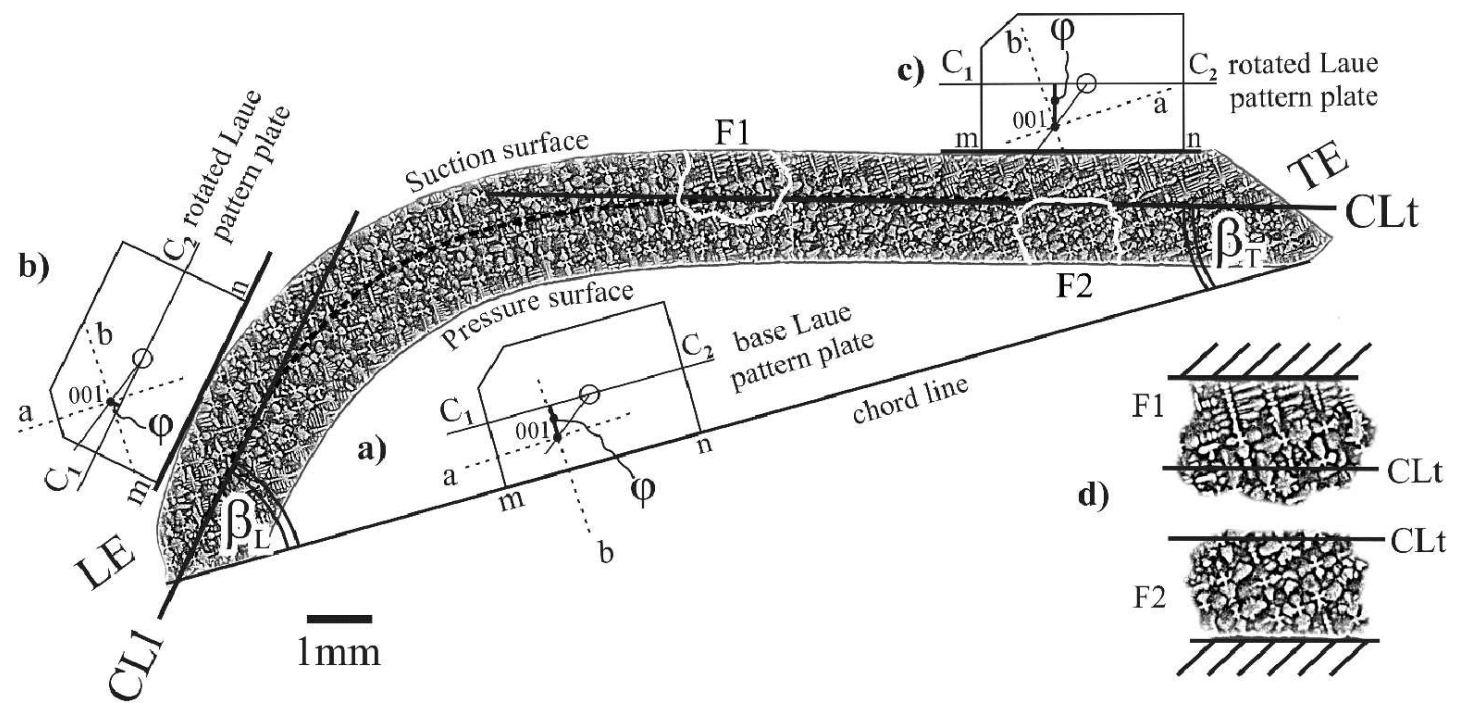

Fig. 2. Example of (a) SEM macro-image of sample surface with base Laue pattern plate, (b, c) rotated Laue pattern plates, (d) fragments of micrograph obtained near the suction (F1) and pressure (F2) surfaces, CLl, CLt - traces of planes parallel to LE, TE (respectively), a, b - directions of dendrite arms, $\mathrm{m}-\mathrm{n}$ - bottom edge of base Laue pattern plate, $\beta_{L}, \beta_{T}$ - angles between traces of planes CLl, CLt (respectively) and $\mathrm{C}_{1}-\mathrm{C}_{2}$ reference line, parallel to bottom edge of Laue pattern plate. Samples surface was prepared from the side indicated by thick arrow (Fig. 1a), withdrawal rate: $5 \mathrm{~mm} / \mathrm{min}$.

The CLl and CLt lines are traces of planes which are parallel to suction (and pressure) surface near the LE and TE, respectively. The $\beta_{L}$ and $\beta_{T}$ are angles between CLl and CLt (respectively) and chord line, which is parallel to the bottom edge $(m-n$, Fig. $2 a)$ of the Laue pattern plate.

The Laue pattern presented in Fig. 3a shows the arrangement of the Laue spots with symmetry of cubic crystal system. The $\alpha$ is the angle between $[001]_{\gamma^{\prime}}$ direction and direction $Z$. Using the base Laue patterns the $\alpha$ angle of dendrites in each sections, near LE and TE areas was determined. To determine the inclination angle of the dendrite cores relative to the mould surface near the LE and TE, transformation of base Laue pattern was performed by rotating base Laue pattern plate $\left(\mathrm{m}-\mathrm{n}\right.$ line) through $\beta_{L}$ angle (anticlockwise) and through $\beta_{L}$ angle (clockwise) (Fig. 2b,c). The $\varphi$ angle (Fig. 2b,c) determines the value of dendrite cores inclination in relation to mould wall surface. If 001 reflection on the rotated Laue patterns (Fig. 2b,c) would be placed above the $\mathrm{C}_{1}-\mathrm{C}_{2}$ line (Fig. 2b), it would mean that dendrite cores are inclined toward the suction surface. Otherwise (001 reflection placed below the $\mathrm{C}_{1}-\mathrm{C}_{2}$ line), dendrite cores will be inclined toward the pressure surface.

The microstructure of analysed samples is not homogeneous in the whole section of the blades airfoil. Dif-

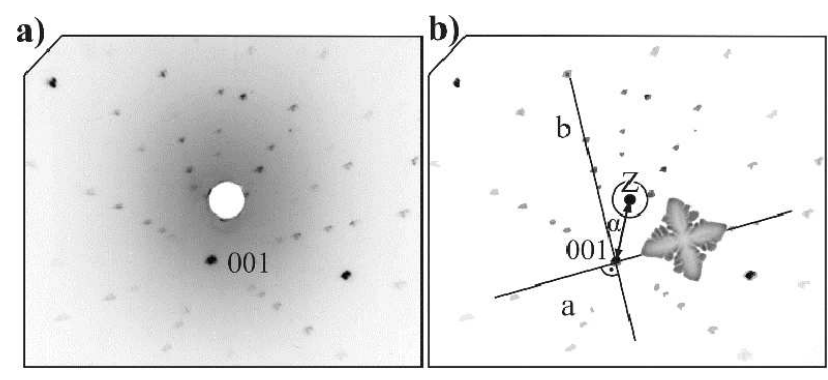

Fig. 3. (a) Example of base Laue pattern and (b) scheme of dendrites arms arrangement.

ferences in size and arrangement of dendrite arms are visible in different areas and airfoils sections of blades obtained with different withdrawal rates. Figures $4 \div 8$ present SEM images of selected fragments of dendritic structure, typical for areas located near the LE and TE, for different sections and withdrawal rates.

Figure 4 shows fragments of SEM macro-image shown in Fig. 2, located near the LE (Fig. 4a) and near the TE (Fig. $4 \mathrm{~b}$ ) of section 3 for blade produced with withdrawal rate of $5 \mathrm{~mm} / \mathrm{min}$. In presented fragments of microstructure the characteristic change of dendrite morphology are visible (indicated by dashed lines). This effect occurs 


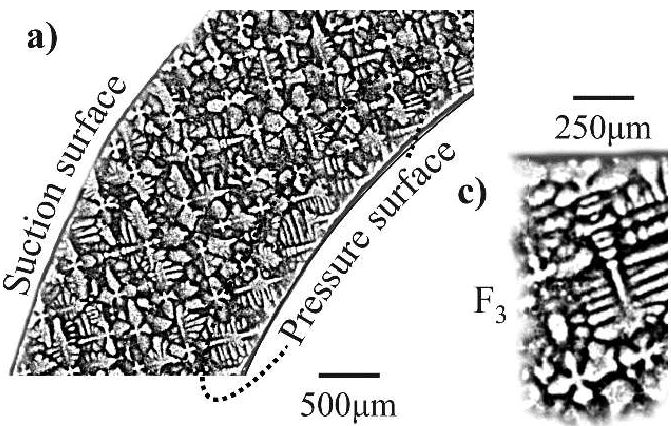

b)

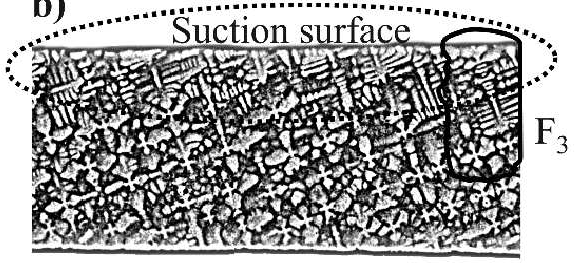

Pressure surface

Fig. 4. SEM (BSE) image of dendritic structure fragment, near the (a) LE and (b) TE; (c) enlarged fragment of micrograph obtained near the suction surface

$\left(\mathrm{F}_{3}\right)$. Section 3 , withdrawal rate: $5 \mathrm{~mm} / \mathrm{min}$.

near the pressure surface of the airfoil for area close to the LE and near the suction surface for area close to the TE, where the "tree-like" morphology occurs (Fig. 4c).

Figure 5 shows fragments of dendritic structure of blades airfoil, located near the LE (Fig. 5a) and near the TE (Fig. 5b) of section 1 for blades produced with withdrawal rate of $4 \mathrm{~mm} / \mathrm{min}$. In presented fragments of microstructure the above-mentioned effect (area of dashed lines) occurs only for area close to the TE near the suction surface of the airfoil.

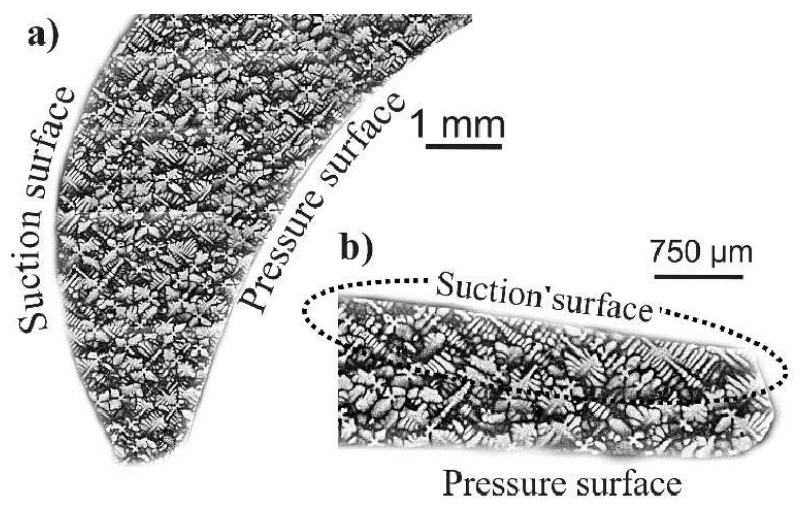

Fig. 5. SEM (BSE) image of dendritic structure fragment, near the (a) LE and (b) TE. Section 1, withdrawal rate: $4 \mathrm{~mm} / \mathrm{min}$.

Figure 6 shows fragments of blades airfoil dendritic structure, located near the LE (Fig. 6a) and near the TE (Fig. 6b) of section 2 or 3 for blades produced with withdrawal rate of $3 \mathrm{~mm} / \mathrm{min}$. In presented fragments of microstructure the effect (areas of dashed lines) occurs only for area close to the TE near the pressure surface of the airfoil.

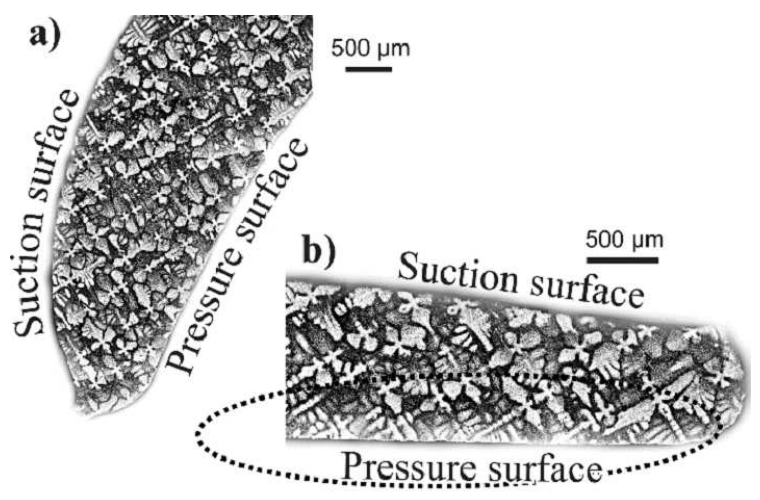

Fig. 6. SEM image of dendritic structure fragment, near the (a) LE and (b) TE. Section 2, withdrawal rate: $3 \mathrm{~mm} / \mathrm{min}$.

Figure 7 shows fragments of blades airfoil dendritic structure, located near the LE (Fig. 7a) and near the TE (Fig. 7b) of section 2 for blades produced with withdrawal rate of $1 \mathrm{~mm} / \mathrm{min}$. In presented fragments of microstructure the effect does not exist.

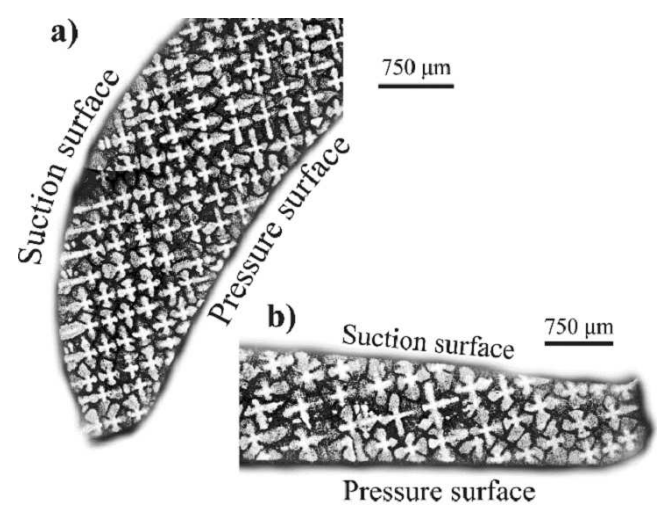

Fig. 7. SEM image of dendritic structure fragment, near the (a) LE and (b) TE. Section 4, withdrawal rate $1 \mathrm{~mm} / \mathrm{min}$.

Comparing of the occurrence of the effect in relation to different sections, section fragments and withdrawal rates allows to deduce that the above effect depends on the inclination of mould walls and occurs more frequently near the TE. Influence of the mould walls on the dendritic morphology, may be dependent on the walls inclination angle to the dendrite cores and on the size of the dendrite arms (related to the withdrawal rate). The change of dendrites morphology close to certain surfaces of mould may be caused by local changes in the dendrites growth rate.

The $\gamma$ angle between the dendrite cores and the walls of the mould for areas near the CLl and CLt were calcu- 
lated by comparing the value and direction of inclination of dendrite cores ( $\varphi$ angle, Fig. 2b) and value and direction of inclination of mould walls near the LE and TE $\left(\delta^{T}, \delta^{L}\right.$ angles, Fig. 1) in relation to the $Z$ axis. The value of $\gamma$ angle gives formulae:

$$
\gamma_{\mathrm{CLl}}=\delta^{L} \pm \varphi_{\mathrm{CLl}}, \quad \gamma_{\mathrm{CLt}}=\delta^{T} \pm \varphi_{\mathrm{CLt}} .
$$

Above calculations show that the previously described effect in the dendrites morphology occurs only in the areas where the inclination of mould wall confines the dendrite cores growth (Fig. 8).

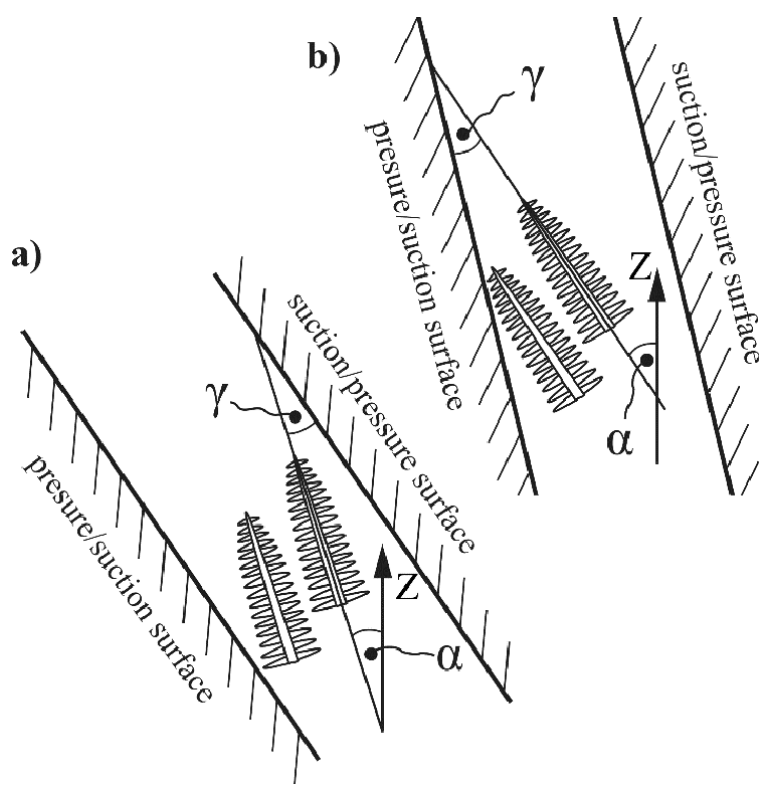

Fig. 8. Scheme of the dendrite cores orientation in relation to different mould walls inclination.

\section{Conclusions}

The effect of change of the dendrites morphology near the mould walls occurs in the areas where the inclination of walls confine the dendrite cores growth.

The above effect occurs more frequently in the thinwalled area, near the trailing edge, where the mould walls strongly influence the microstructure.

\section{Acknowledgments}

The research was supported by the Polish National Centre for Research and Development under Grant No. INOTECH K2/IN2/57/182858/NCBR/13.

\section{References}

[1] R. Reed, The Superalloys: Fundamentals and Application, Cambridge University Press, Cambridge 2006.

[2] M.J. Donachie, S.J. Donachie, Superalloys. A Technical Guide, ASM International, Ohio 2002.

[3] A. Wawrzyniak, M. Wieczorek, T. Ryniewicz, Przeglad mechaniczny 12, 14 (2012) (in Polish).

[4] J.W. Aveson, G. Reinhart, H. Nguyen-Thi, N. Mangelinck-Noël, A. Tandjaoui, B. Billia, K. Goodwin, T.A. Lafford, J. Baruchel, H.J. Stone, N. D'Souza, in: Superalloys 2012, Eds. E.S. Huron, R.C. Reed, M.C. Hardy, M.J. Mills, R.E. Montero, P.D. Portella, J. Telesman, TMS, 2012, p. 615.

[5] E. Rzyankina, D. Szeliga, N. Mahomed, A. Nowotnik, Appl. Mech. Mater. 372, 54 (2013).

[6] A. Onyszko, W. Bogdanowicz, K. Kubiak, J. Sieniawski, Cryst. Res. Technol. 45, 1326 (2010). 